Check for updates

Cite this: Mater. Adv., 2020, 1,2332

Received 11th June 2020, Accepted 18th August 2020

DOI: $10.1039 / \mathrm{d} 0 \mathrm{ma00408a}$

rsc.li/materials-advances

\section{The wet chemical synthesis of surfactant-capped quasi-spherical silver nanoparticles with enhanced antibacterial activity $\dagger$}

\author{
Marryam Mahmood, ${ }^{a}$ Mehwish Abid, ${ }^{a}$ Muhammad Faizan Nazar, (D) *a \\ Muhammad Nadeem Zafar, (D) *a Muhammad Asam Raza, ${ }^{a}$ Muhammad Ashfaq, ${ }^{2}$ \\ Asad Muhammad Khan, ${ }^{b}$ Sajjad Hussain Sumrra and Muhammad Zubair ${ }^{a}$
}

Capped silver nanoparticles (AgNPs) are used in many applications in the biomedical field because of their unique structural flexibility. Herein, a viable chemical reduction synthesis method for AgNPs is described employing ionic surfactants as stabilizing as well as capping agents. Stabilized AgNPs of a quasi-spherical shape were obtained using silver nitrate $\left(\mathrm{AgNO}_{3}\right)$ as a precursor and sodium borohydride $\left(\mathrm{NaBH}_{4}\right)$ as a reducing agent under multiple ionic micellar hierarchical approaches. UV-Visible spectroscopy studies showed characteristic single and sharp absorbance peaks in the range 400 to $420 \mathrm{~nm}$, which indicates the absence of the self-aggregation of AgNPs, whereas the FTIR results indicated the surfactant functionalities responsible for the stability of the AgNPs. Micrographs of the as-obtained AgNPs, obtained via electron microscopy (SEM, STEM), demonstrated a fine monomodal collection of spherical nanoparticles of $<15 \mathrm{~nm}$, which was further confirmed by dynamic light scattering (DLS) measurements. Moreover, the stability of the as-synthesized AgNPs was evaluated by determining the charge present on the surface, i.e., positive or negative, indicating the stability of the monodispersed AgNPs against flocculation. The bactericidal ability of the surfactant capped AgNPs was checked by measuring the zone of inhibition using the agar-well diffusion method. The results indicated that CTAB-AgNPs and SDS-AgNPs showed enhanced bactericidal effects, whereas DDAB-AgNPs, DOSSAgNPs, and MES-AgNPs showed no significant activity against multidrug-resistant strains of bacteria.

\section{Introduction}

Since ancient times, metal has attracted great interest as an ornament or jewellery. In contrast, the nanostructures of these metals have been of interest due to their larger surface area to volume ratio, size, shape, and electrical properties, especially in the field of biomedicine. Due to significant size-related perspectives, various metal nanoparticles (NPs) are being synthesized and adopted for use. ${ }^{1,2}$ Among the precious metal nanoparticles, silver nanoparticles (AgNPs) have become the focus of scientific attention because they have the ability to interact with various biomolecules against a broad spectrum of

\footnotetext{
${ }^{a}$ Department of Chemistry, University of Gujrat, Gujrat, 50700, Pakistan. E-mail: znadeempk@gmail.com,nadeem.zafar@uog.edu.pk, faizan_qau@yahoo.com, faizan.nazar@uog.edu.pk

${ }^{b}$ Department of Chemistry, COMSATS Institute of Information Technology, Abbottabad, 22060, Pakistan

$\dagger$ Electronic supplementary information (ESI) available: FTIR spectra and basic molecular structures of the pure surfactants, SEM results from DDAB-AgNPs, DOSS-AgNPs, and MES-AgNPs, and antibacterial activity results. See DOI: 10.1039/ d0ma00408a
}

bacteria and because of their fungicidal activity. ${ }^{3}$ Although scientists are fascinated by AgNPs due to their multifunctional properties and their widespread uses in electronics, ${ }^{4}$ the textile industry, ${ }^{5,6}$ waste water treatment, ${ }^{7}$ as potent disinfectants, ${ }^{8}$ and dentistry, ${ }^{9}$ however, expansion of multidrug-resistant bacteria, being an alarming situation, has shifted the scientists' attention to the use of AgNPs in biomedical applications. ${ }^{3}$

The AgNPs are being synthesized, physically and/or chemically, using multiple stabilizers with various protocols, i.e., hydrothermal with nanocellulose ${ }^{10}$ chemical reduction and stabilization by dextran, ${ }^{11}$ and photochemical in the presence of collagen. ${ }^{12}$ Despite all this, the stability of the NPs have been a great concern which is related to the synthesis of AgNPs, because of the expense and the harmful environmental impacts of them being employed as stabilizers. ${ }^{13}$

Surfactants, and amphiphilic molecules, primarily function as stabilizers and provide colloidal stability and prevent agglomeration and excessive growth. In addition, it was also found that these amphiphilic molecules acted as optimal capping agents, thereby greatly improving the antibacterial activity of the modified AgNPs. However, it is also evident that 
the bactericidal and fungicidal properties are morphologically dependent on the AgNPs. Fortunately, surfactants can be control the morphology of colloidal NPs. ${ }^{14-16}$ Although various silver nanostructures such as silver nanorods, nanoplates, and triangular shapes have been reported to enhance bactericidal properties, conversely it has been concluded from previous reports in the literature that spherical AgNPs have a poorer bactericidal performance compared to other shapes and morphologies. ${ }^{14,17,18}$

In the context of differential morphological dependent bactericidal properties of AgNPs, the current work describes the development of quasi-spherical AgNPs via a one-pot wet chemical reduction route under multiple ionic micellar hierarchical approaches. The purpose is to enhance the bactericidal efficiency of spherical AgNPs. Different capping agents i.e., cetyltrimethylammonium bromide (CTAB), di-n-dodecyldimethylammonium bromide (DDAB), sodium dodecyl sulfate (SDS), dioctyl sodium succinate (DOSS), 2-mercaptoethanesulfonate sodium (MES) are used (basic molecular structures are given in Table S1 (ESI $\dagger$ )). The characterization of the synthesized AgNPs was accomplished using UV-Visible, and Fourier transform infrared (FTIR) spectroscopies, DLS, and zeta potential measurements, whereas the morphology of AgNPs was determined using scanning electron microscopy (SEM) and scanning transmission electron microscopy (STEM). The bactericidal activity against nine bacterial strains was checked using the agar-well diffusion method, and the zone of inhibition (ZOI) was determined accordingly. Based on the data provided in this study, it was found that spherical AgNPs show enhanced bactericidal efficiency when capped with mono-alkyl ionic surfactants compared to capping with the di-alkyl surfactant moiety.

\section{Experimental section}

\section{Materials and chemicals}

Silver nitrate $\left(\mathrm{AgNO}_{3}, 99 \%\right)$ and sodium borohydride $\left(\mathrm{NaBH}_{4}\right.$, $\geq 98 \%$ ) were purchased from VWR Chemicals. Cetyltrimethylammonium bromide (CTAB, $\geq 99 \%$ ) was purchased from VWR, di- $n$-dodecyldimethylammonium bromide (DDAB, 98\%) from Alfa Aesar, sodium dodecyl sulfate (SDS, $\geq 99 \%$ ) from Duksan Pure Chemicals, dioctyl sodium succinate (DOSS, 96\%) and 2-mercaptoethanesulfonate sodium (MES) from Acros organics. All the chemicals were of analytical grade and used as received without any further modifications. Double distilled $\mathrm{H}_{2} \mathrm{O}$ was used for the preparation of all the solutions.

\section{Synthesis of capped-AgNPs}

For the synthesis of AgNPs, $\mathrm{AgNO}_{3}$ was used as the precursor of silver ions, $\mathrm{NaBH}_{4}$ was used as the reducing agent, and different surfactants were utilized as capping agents. A portion $(2.0 \mathrm{~mL})$ of $\mathrm{AgNO}_{3}(1 \mathrm{wt} \%)$ was diluted to $100 \mathrm{~mL}$ with distilled water. Then the optimal amount of each capping agent was prepared in $100 \mathrm{~mL}$ of distilled water. The $\mathrm{AgNO}_{3}$ and capping agent (one per sample) solutions were then mixed together and continuously stirred at room temperature, maintaining the concentration of each surfactant, i.e., CTAB (2.0 mM), DDAB

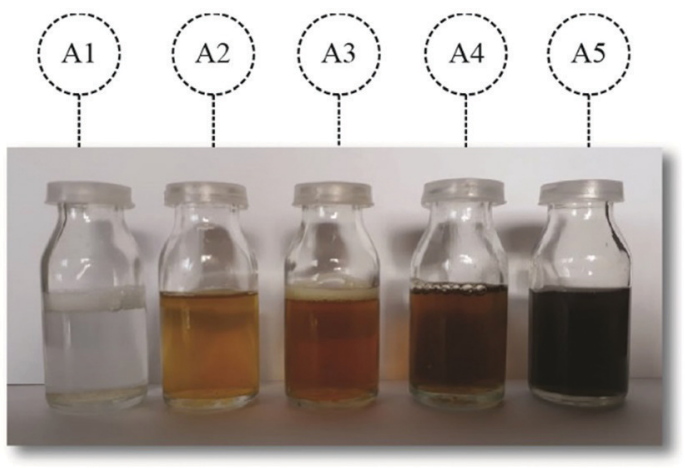

Fig. 1 The visual appearances of various capped-AgNPs.

(0.3 mM), SDS (9.0 mM), DOSS (3.0 mM) and MES (1.0 mM) above their respective critical micelle concentration (CMC) values. After $10 \mathrm{~min}$, freshly prepared $\mathrm{NaBH}_{4}$ solution $(0.04 \mathrm{~g}$ in $1 \mathrm{~mL}$ of $\mathrm{H}_{2} \mathrm{O}$ ) was added dropwise, resulting in the formation of capped-AgNPs. After all the $\mathrm{NaBH}_{4}$ solution was added, the reaction mixture was stirred overnight. At the end of the reaction, a brownish colloidal solution was obtained, as shown in Fig. 1. The as-synthesized capped-AgNPs were labelled as A1, A2, A3, A4 and A5 for CTAB-AgNPs, DDAB-AgNPs, SDS-AgNPs, DOSS-AgNPs and MES-AgNPs, respectively.

\section{Characterization of capped-AgNPs}

Spectroscopic measurements. The IR spectra of $\mathrm{AgNO}_{3}$, pure surfactant solutions and as-synthesized capped-AgNPs at a frequency range of 400-4000 $\mathrm{cm}^{-1}$ were observed with a Brucker Alpha series FTIR spectrometer. The characteristic plasmonic peaks of the synthesized AgNPs were measured by UV-Visible spectroscopy.

Particle size analysis. A Malvern Instruments, Nano ZSP Zetasizer was used to record the average particle size, polydispersity index (PDI) and zeta potential of the capped-AgNPs. At a temperature of $25{ }^{\circ} \mathrm{C}$, a clear disposable zeta cell was used to measure the size distributions of samples with a laser having a wavelength of $635 \mathrm{~nm}$.

Morphology of capped-AgNPs. The structural information of the capped-AgNPs was assessed using a $15 \mathrm{kV}$ FEI, Nova NanoSEM 450 SEM equipped with an A-B mode STEM detector. The sample was restricted by dripping it onto a carbon-coated copper mesh and air-drying. After coating with a $1 \%$ ammonium molybdate solution, it had swollen and was then dried for $10 \mathrm{~s}$. It was further dried before the microscopic studies.

\section{Antibacterial activity}

The antibacterial activity of the synthesized AgNPs was measured by an agar-well diffusion method. A suspension of microorganisms with a concentration of $10^{5}-10^{6}$ colony forming units (CFU) per well was used. The volume of the microbial inoculum was distributed on the surface of the agar plate. A $5 \mathrm{~mm}$ diameter hole in the agar was made with a sterile cork, and a volume of synthetic AgNP dispersion was used as a sample. The culture 
Table 1 Characterisation of the as-synthesized capped AgNPs

\begin{tabular}{|c|c|c|c|c|c|c|}
\hline Capping agent & $\lambda_{\max }(\mathrm{nm})$ & $\varepsilon\left(\mathrm{dm}^{3} \mathrm{~mol}^{-1} \mathrm{~cm}^{-1}\right) \times 10^{8}$ & $\begin{array}{l}\text { Concentration } \\
\left(\mathrm{mol} \mathrm{dm}^{-3}\right) \times 10^{-12}\end{array}$ & Particle size (nm) & $\zeta$-Potential $(\mathrm{mV})$ & PDI \\
\hline CTAB & 415 & 336.0 & 3.0050 & $5.50 \pm 0.50$ & 16.8 & 0.183 \\
\hline DDAB & 406 & 145.0 & 116.55 & $9.20 \pm 0.20$ & 31.6 & 0.216 \\
\hline SDS & 410 & 240.5 & 101.12 & $6.70 \pm 0.30$ & -33.1 & 0.195 \\
\hline DOSS & 414 & 336.0 & 36.690 & $4.50 \pm 0.50$ & -45.2 & 0.203 \\
\hline MES & 406 & 145.0 & 99.240 & $11.30 \pm 0.40$ & -28.5 & 0.225 \\
\hline
\end{tabular}

plate was incubated at $37{ }^{\circ} \mathrm{C}$ for $24 \mathrm{~h}$, and then the ZOI was evaluated visually to check for bacterial activity.

\section{Results and discussion}

\section{UV-visible spectroscopy to measure plasmonic peaks}

The UV-visible spectroscopy was utilized to obtain the absorption spectra of $\mathrm{AgNO}_{3}$ (precursor) and all the as-synthesized cappedAgNPs, shown in Fig. 2(a). Optical and structural information about the samples were also obtained from the electronic spectrum. The $\mathrm{AgNO}_{3}$ showed a characteristic plasmonic peak at $302 \mathrm{~nm} .{ }^{19}$ The sample A1 (slightly yellow), A2 (yellow), A3 (orange), A4 (light brown) and A5 (dark brown) (by eye indistinguishable from the blank shown in Fig. 1), showed maximum absorption at 415, 406, 410, 414 and $406 \mathrm{~nm}$, respectively, as shown in Fig. 2(a). A single plasmonic resonance peak was obtained for each sample, which showed the uniformity of the particles formed and a certain degree of monodispersity. Compared with the absorption of $\mathrm{AgNO}_{3}$, the absorption of each nano-sample was red-shifted, which confirmed the reduction of silver ions and the formation of silver particles. ${ }^{19}$ The concentration of silver particles was calculated using Lambert-Beer's law, and the data obtained is given in Table 1.

\section{Infrared study}

The FTIR spectroscopy was used to observe the presence of functional groups and the changes in the peaks that occurred due to the changes in the nature of the surfactants. The FTIR spectra of $\mathrm{AgNO}_{3}$ (precursor) and all the synthesized, cappedAgNPs are shown in Fig. 2(b). In the spectrum of $\mathrm{AgNO}_{3}$ solution, two peaks were observed at 3223.11 and $1632.44 \mathrm{~cm}^{-1}$. Due to the presence of the aqueous medium, a broad absorption peak was observed at $3221.31 \mathrm{~cm}^{-1}$, which corresponded to $\mathrm{O}-\mathrm{H}$ stretching. The peak at $1632.79 \mathrm{~cm}^{-1}$, corresponded to the stretching of the $\mathrm{N}-\mathrm{O}$ group. In the spectra of the synthesized silver dispersion, the peak at $3223.11 \mathrm{~cm}^{-1}$ became broader, whereas the peak at $1636.79 \mathrm{~cm}^{-1}$ underwent a red shift and a decrease in intensity occurred. In the spectra of A1 and A2, two new peaks appeared at $1081.19 \mathrm{~cm}^{-1}$ and $1083.58 \mathrm{~cm}^{-1}$, respectively, corresponding to $\mathrm{C}-\mathrm{N}$ stretching. In the case of A3, A4 and A5, new peaks appeared at $1084.60,1087.22$ and $1093.83 \mathrm{~cm}^{-1}$, respectively, which corresponded to the stretching vibration of the $\mathrm{S}=\mathrm{O}$ group. The spectra of the pure surfactant solution are also given in the Fig. S1 (ESI $\dagger$ ), which confirmed the change in the nano-sample spectrum. The broadening, shifting and appearance of new peaks in the spectra of the nano-samples indicated an interaction between the surfactant and surface of the silver particles. ${ }^{20}$
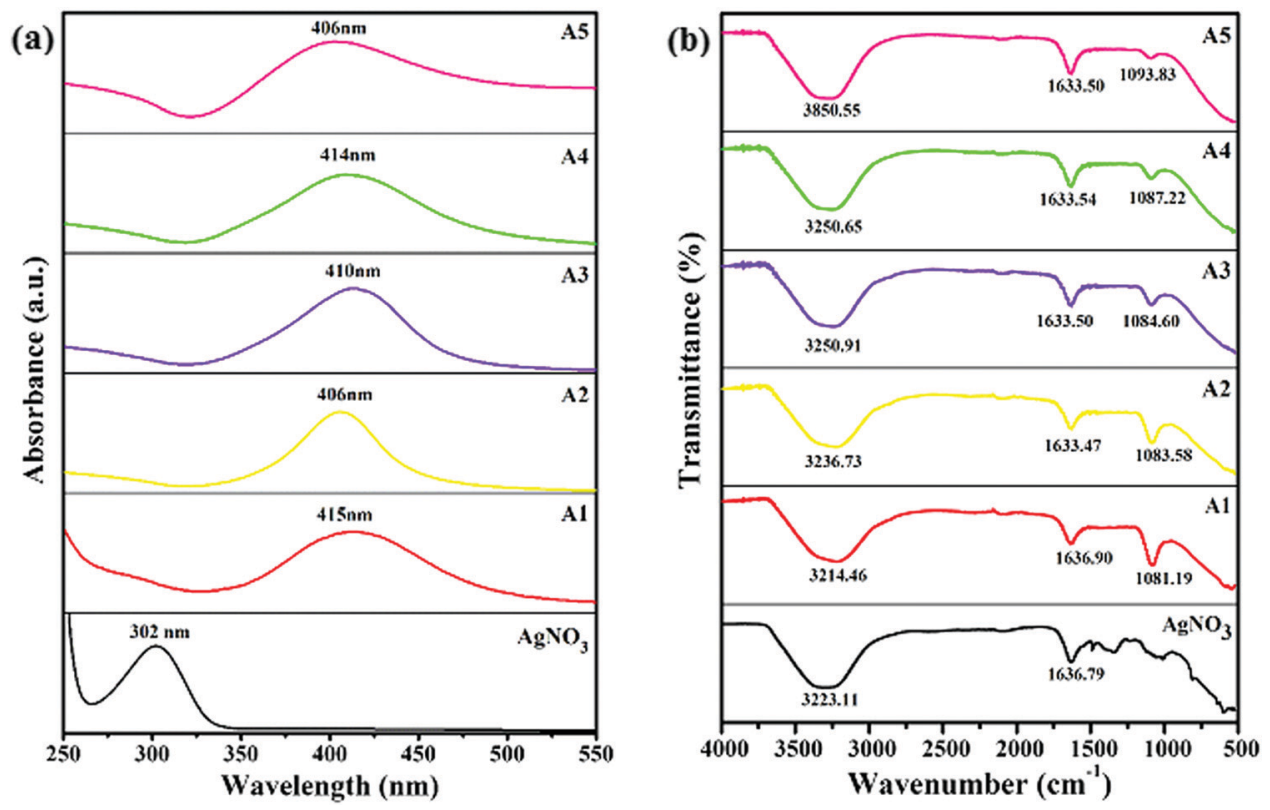

Fig. 2 UV-visible (a) and FTIR (b) spectra for $\mathrm{AgNO}_{3}$ and capped-AgNPs. 

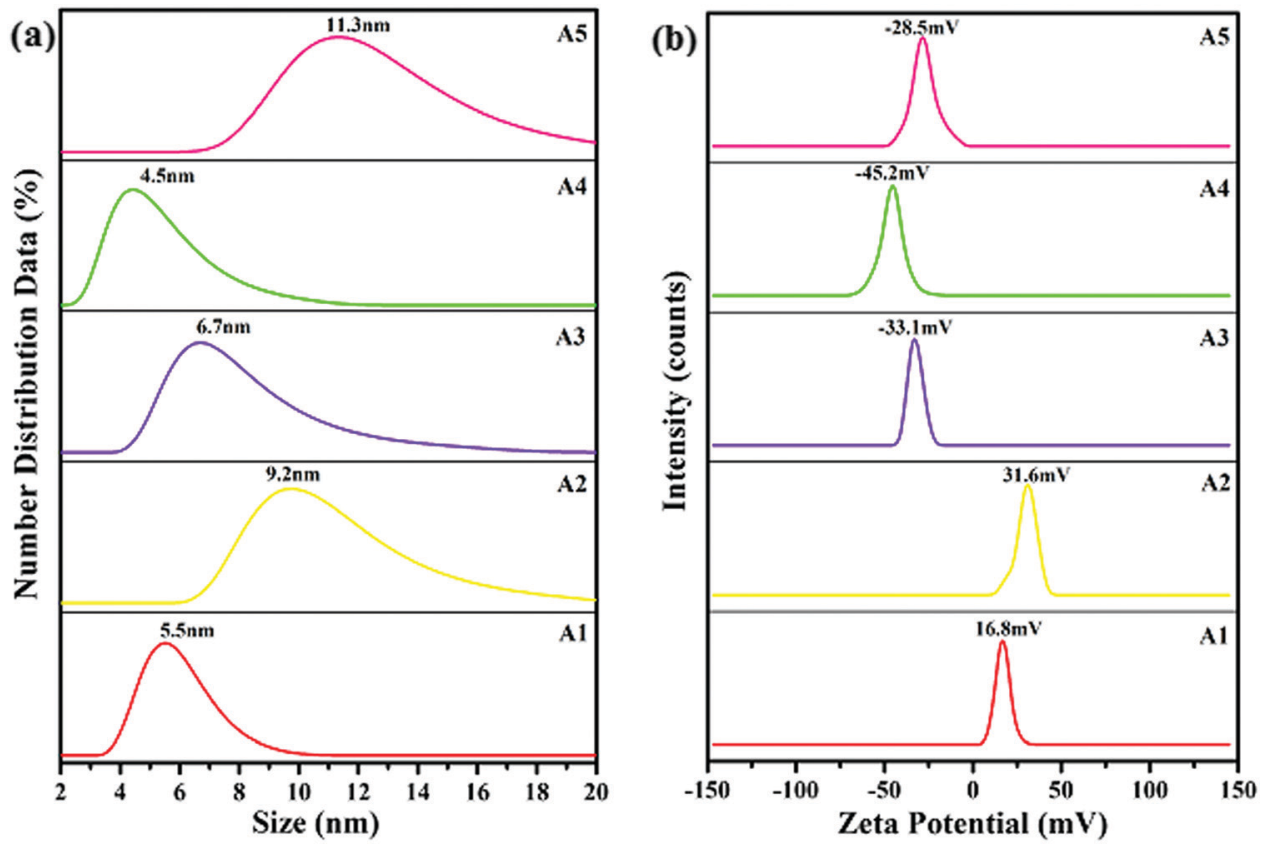

Fig. 3 Particle size distributions (a) and zeta potentials (b) of surfactant capped AgNPs.

\section{Dynamic light scattering study}

The average particle size of the synthesized AgNPs was determined by a DLS technique. From the results, it was confirmed that the sizes of the silver particles formed were in the nanometer range of 4-12 $\mathrm{nm}$. The formation and capping of the silver particles had already been confirmed by the results of the UV-visible and FTIR techniques, respectively. As shown in Fig. 3(a), in the presence of the capping agents CTAB, DDAB, SDS, DOSS and MES, the average particle size of the AgNPs is $5.5 \pm 0.50,9.20 \pm 0.20,6.70 \pm 0.30,4.50 \pm 0.50$ and $11.30 \pm 0.30 \mathrm{~nm}$, respectively.

\section{Zeta potential}

The zeta potentials of the synthesized AgNPs: A1, A2, A3, A4 and A5 are plotted in Fig. 3(b). The surfactants were adsorbed on to the surface of AgNPs through a hydrophobic interaction between the hydrophobic groups of the surfactant and the negative surface of the AgNPs. As reported earlier, the stability of the AgNPs increased with the surface charge of AgNPs due to the presence of surfactants. ${ }^{21}$ Anionic surfactants generated a negative charge, whereas the cationic surfactants generated a positive charge on the surface of the AgNPs. ${ }^{22}$ The zeta potentials of the nano-samples: A1, A2, A3, A4 and A5 were found to be: $+16.8,+31.6,-33.1,-45.2$ and $-28.5 \mathrm{mV}$, respectively. The zeta potential, and hence the stability of the synthesized AgNPs in the presence of the capping agent decreased in the order: DOSS $>$ DDAB $>$ SDS $>$ MES $>$ CTAB. Compared with DDAB, the AgNPs had a high stability when DOSS was present as a dialkyl chain surfactant. Whereas, for the single alkyl chain, SDS was a more suitable surfactant when compared to CTAB and MES, because the larger the charge value appearing on the surface of the AgNPs the greater the stability of the particle. ${ }^{21}$

\section{Scanning transmission electron microscopy}

The morphological information about the synthesized AgNPs was obtained from the STEM and SEM micrographs, as shown in Fig. $4(a, b)$ and $\left(a^{\prime}, b^{\prime}\right)$, respectively. The photomicrographs
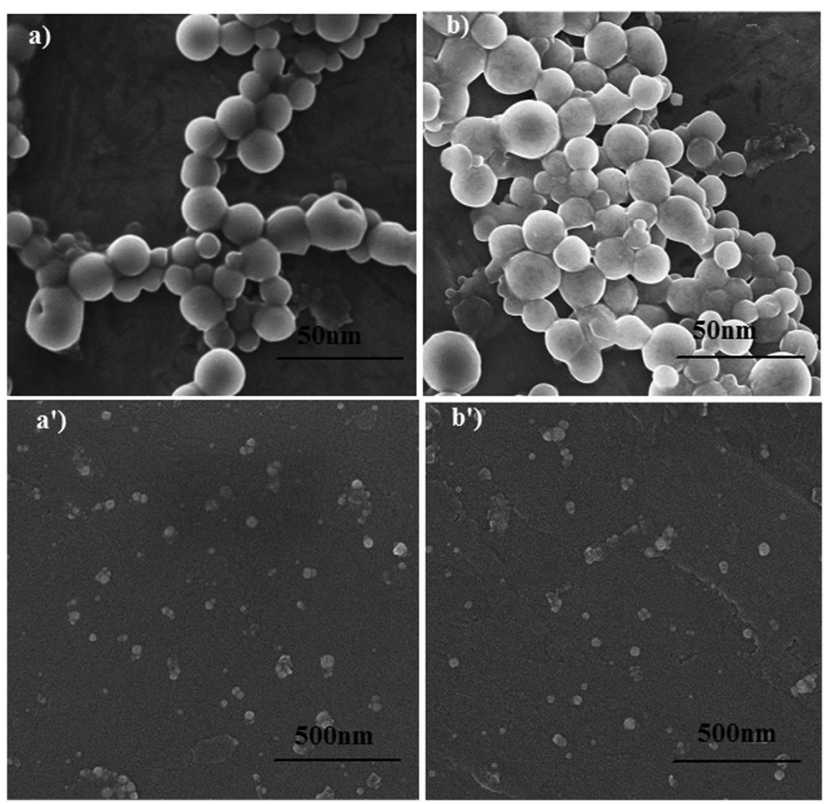

Fig. 4 STEM ( $a$ and $b$ ) and SEM ( $a^{\prime}$ and $b^{\prime}$ ) micrographs of CTAB-AgNPs (A1) ( $a$ and $a^{\prime}$ ) and SDS-AgNPs (A3) (b and $b^{\prime}$ ). 

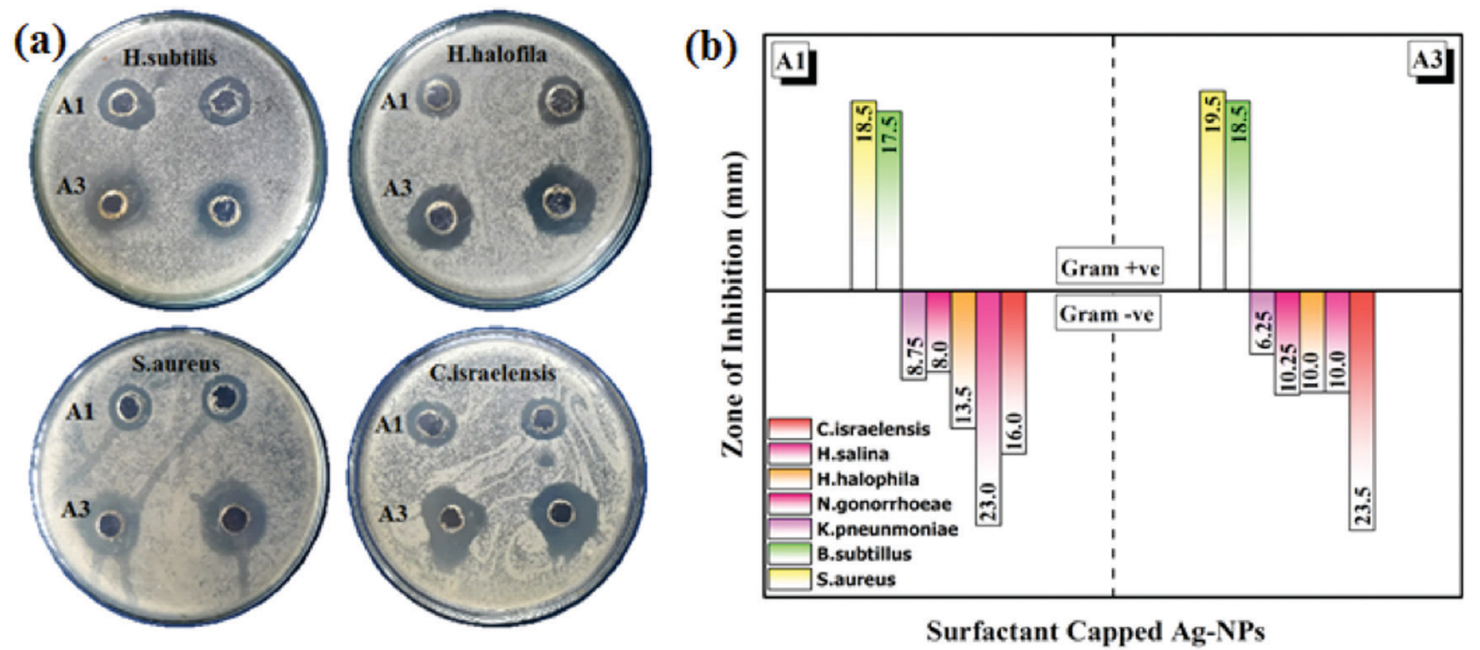

Fig. 5 The antibacterial activities of CTAB-AgNPs (A1) and SDS-AgNPs (A3): (a) zone of inhibition against B. subtilis, H. halofila, S. aureus, and $C$. israelensis; and (b) comparative results of the zone of inhibition against Gram-positive and Gram-negative bacteria.

confirm that the synthesized surfactant capped-AgNPs (CTABAgNPs and SDS-AgNPs) were quasi-spherical and monodispersed in nature. The SEM micrographs of the DDAB-AgNPs, DOSS-AgNPs and MES-AgNPs are given in Fig. S2 (ESI $\dagger$ ), which depict the quasi-spherical shape and monodispersity of the synthesized NPs.

\section{Antibacterial activity}

The in vitro antibacterial activity of the synthesized AgNPs was tested against nine (9) strains of bacteria: Staphylococcus aureus (S. aureus), Bacillus subtilis (B. subtilis), Klebsiella pneumoniae (K. pneumoniae), Neisseria gonorrhoeae ( $N$. gonorrhoeae), Halomonas halophila ( $H$. halophila), Halomonas salina (H. salina), Chromohalobacter israelensis (C. israelensis), Shigella sonnei (S. sonnei) and Escherichia coli (E. coli). Table S2 (ESI $\dagger$ ) shows the measurements of the ZOIs for all the nano-samples with a positive control (Streptomycin). The size of the sample taken was $30 \mu \mathrm{L}$. It was observed that samples A1 and A3 showed activity against seven (7) bacterial strains as shown in the Fig. 5 . Whereas, samples A2, A4 and A5 did not show any significant bactericidal properties against the tested bacterial strains. It can be seen that CTAB-AgNPs and SDS-AgNP were more active against Gram-negative bacteria than Gram-positive bacteria. This can be explained by differences in the peptidoglycan thickness in the bacterial cell wall (Fig. 5a). Compared to Grampositive bacteria, Gram-negative bacteria have thinner cell walls, as reported previously. ${ }^{23}$ Thus, the surfactant-capped quasispherical AgNPs obtained have bactericidal properties due to the fact that they interact with bacterial cells and cause their destruction. According to previous reports, they affect the normal function of bacterial cells by reacting with soft alkali and interacting with respiratory enzymes, interact with bacterial cells, inhibit bacterial growth, and change bacterial DNA. ${ }^{24,25}$

Although AgNPs exhibit antimicrobial properties compared to other precious metal NPs, this ability is entirely dependent on size and morphology of the particles. It can be confirmed from the comparative literature (listed in Table $2^{26-32}$ ) that spherical AgNPs have a lower bactericidal performance with different methods in the presence of different stabilizers and capping agents. However, in the current work, using surfactants as capping agents, especially monoalkyl chain ionic surfactants (i.e., CTAB and SDS), the bactericidal properties

Table 2 Comparison of spherical AgNPs synthesized via various methodologies with variable size having bactericidal abilities against Gram-positive and Gram-negative bacterial strains

\begin{tabular}{|c|c|c|c|c|c|c|}
\hline \multirow[b]{2}{*}{ Reducing agent } & \multirow[b]{2}{*}{ Stabilizing/capping agent } & \multirow[b]{2}{*}{ Shape } & \multirow[b]{2}{*}{ Size (nm) } & \multicolumn{2}{|c|}{ Zone of inhibition $(\mathrm{mm})$} & \multirow[b]{2}{*}{ Ref. } \\
\hline & & & & Gram +ve & Gram -ve & \\
\hline \multicolumn{2}{|c|}{ Beetroot extract } & Spherical & 15 & 19.00 & 7.000 & 26 \\
\hline \multicolumn{2}{|c|}{ Candida albicans (fungus) } & Spherical & $60.88-65.57$ & 14.00 & 16.00 & 27 \\
\hline \multicolumn{2}{|c|}{$\begin{array}{l}\text { Phlomis (plant extract) } \\
\text { Panax ginseng (leaves extract) }\end{array}$} & Predominantly spherical & 25 & 14.70 & 14.90 & 28 \\
\hline \multicolumn{2}{|c|}{ Panax ginseng (leaves extract) } & Spherical & $5-15$ & 16.00 & 14.00 & 29 \\
\hline $\mathrm{NaBH}_{4}$ & Piper betle (leaf extract) polyaniline & Spherical & $10-30$ & 32.78 & 27.12 & 30 \\
\hline $\mathrm{NaBH}_{4}$ & PVP & Spherical & $15-50$ & - & 1.500 & 17 \\
\hline \multicolumn{2}{|c|}{ Carboxymethyl cellulose } & Spherical & $5-15$ & 28.23 & 30.13 & 31 \\
\hline \multicolumn{2}{|c|}{ Bacillus brevis (bacterial strain) } & Spherical & $41-68$ & 15.00 & 17.00 & 32 \\
\hline $\mathrm{NaBH}_{4}$ & Starch & Spherical & $8 \pm 4$ & 7.000 & 7.700 & 23 \\
\hline \multirow[t]{2}{*}{$\mathrm{NaBH}_{4}$} & CTAB & Quasi-spherical & $5.50 \pm 0.50$ & 18.50 & 16.00 & This study \\
\hline & SDS & & $6.70 \pm 0.30$ & 19.50 & 23.50 & \\
\hline
\end{tabular}


of spherical AgNPs have been significantly enhanced, as shown in Fig. 5.

\section{Conclusions}

A one-pot, wet chemical reduction method was used to synthesize monomodal, quasi-spherical surfactant-capped AgNPs with an average diameter of $<15 \mathrm{~nm}$. The comparative stability and bactericidal ability of capped-AgNPs were determined using their zeta potentials and inhibition zone values, respectively. The results show that among the di-alkyl chain surfactants, DOSS $(-45.2 \mathrm{mV})$ is more suitable than DDAB (+31.6 mV), whereas among the mono-alkyl chain surfactants, SDS $(-33.1 \mathrm{mV})$ is more suitable than CTAB $(+16.8 \mathrm{mV})$ and MES (-28.5 mV). Compared to dialkyl chain surfactants, monoalkyl chain surfactants have stronger bactericidal capabilities. Conversely, CTAB-AgNPs and SDS-AgNPs showed significant activity against $S$. aureus (Gram-positive strain), with ZOIs of 18.5 and $19.5 \mathrm{~mm}$, respectively, whereas against Gram-negative strains, CTAB-AgNPs showed significant activity (ZOI of $23.0 \mathrm{~mm}$ ) towards $H$. salina, and SDS-AgNPs showed activity towards C. israelensis, with a ZOI of $23.5 \mathrm{~mm}$. Although DOSS is more suitable for the stability of the AgNPs, in contrast, AgNPs capped with SDS and CTAB have a greater ability to inhibit bacterial growth. Above all, the analyses show that surfactants play a key role in enhancing the stability and bactericidal capabilities of spherical AgNPs.

\section{Conflicts of interest}

There are no conflicts to declare.

\section{Acknowledgements}

The authors wish to express their gratitude to the Department of Chemistry, University of Gujrat, Pakistan, for providing laboratory facilities. The authors acknowledge financial support from the Higher Education Commission of Pakistan through an NRPU project (20-4557/NRPU/R\&D/HEC/14/481).

\section{Notes and references}

1 M. Hoseinnejad, S. M. Jafari and I. Katouzian, Inorganic and metal nanoparticles and their antimicrobial activity in food packaging applications, Crit. Rev. Microbiol., 2018, 44, 161-181.

2 Y. Zhou, C. Jin, Y. Li and W. Shen, Dynamic behavior of metal nanoparticles for catalysis, Nano Today, 2018, 20, 101-120.

3 A. C. Burdusel, O. Gherasim, A. M. Grumezescu, L. Mogoanta, A. Ficai and E. Andronescu, Biomedical applications of silver nanoparticles: an up-to-date overview, Nanomaterials, 2018, 8, 681.
4 Z. Zhang, X. Zhang, Z. Xin, M. Deng, Y. Wen and Y. Song, Synthesis of monodisperse silver nanoparticles for ink-jet printed flexible electronics, Nanotechnology, 2011, 22, 425601.

5 C. H. Xue, J. Chen, W. Yin, S. T. Jia and J. Z. Ma, Superhydrophobic conductive textiles with antibacterial property by coating fibers with silver nanoparticles, Appl. Surf. Sci., 2012, 258, 2468-2472.

6 F. Zhang, X. Wu, Y. Chen and H. Lin, Application of silver nanoparticles to cotton fabric as an antibacterial textile finish, Fibers Polym., 2009, 10, 496-501.

7 T. A. Dankovich and D. G. Gray, Bactericidal paper impregnated with silver nanoparticles for point-of-use water treatment, Environ Sci Technol, 2011, 45, 1992-1998.

8 S. P. Deshmukh, S. M. Patil, S. B. Mullani and S. D. Delekar, Silver nanoparticles as an effective disinfectant: a review, Mater. Sci. Eng., C, 2019, 97, 954-965.

9 R. A. Bapat, T. V. Chaubal, C. P. Joshi, P. R. Bapat, H. Choudhury, M. Pandey, B. Gorain and P. Kesharwani, An overview of application of silver nanoparticles for biomaterials in dentistry, Mater. Sci. Eng. C, 2018, 91, 881-898.

10 X. Zhang, H. Sun, S. Tan, J. Gao, Y. Fu and Z. Liu, Hydrothermal synthesis of $\mathrm{Ag}$ nanoparticles on the nanocellulose and their antibacterial study, Inorg. Chem. Commun., 2019, 100, 44-50.

11 K. P. Bankura, D. Maity, M. M. Mollick, D. Mondal, B. Bhowmick, M. K. Bain, A. Chakraborty, J. Sarkar, K. Acharya and D. Chattopadhyay, Synthesis, characterization and antimicrobial activity of dextran stabilized silver nanoparticles in aqueous medium, Carbohydr. Polym., 2012, 89, 1159-1165.

12 E. I. Alarcon, K. Udekwu, M. Skog, N. L. Pacioni, K. G. Stamplecoskie, M. González-Béjar, N. Polisetti, A. Wickham, A. Richter-Dahlfor, M. Grifitth and J. C. Scaiano, The biocompatibility and antibacterial properties of collagen-stabilized, photochemically prepared silver nanoparticles, Biomaterials, 2012, 33, 4947-4956.

13 S. Skoglund, E. Blomberg, I. O. Wallinder, I. Grillo, J. S. Pedersen and L. M. Bergström, A novel explanation for the enhanced colloidal stability of silver nanoparticles in the presence of an oppositely charged surfactant, Phys. Chem. Chem. Phys., 2017, 19, 28037-28043.

14 L. Kvítek, A. Panáček, J. Soukupová, M. Kolář, R. Večeřová, R. Prucek, M. Holecová and R. Zbořil, Effect of surfactants and polymers on stability and antibacterial activity of silver nanoparticles (NPs), J. Phys. Chem. C, 2008, 112, 5825-5834.

15 I. A. Wani, S. Khatoon, A. Ganguly, J. Ahmed and T. Ahmad, Structural characterization and antimicrobial properties of silver nanoparticles prepared by inverse microemulsion method, Colloids Surf., B, 2013, 101, 243-250.

$16 \mathrm{~W}$. Zhang, X. Qiao and J. Chen, Synthesis and characterization of silver nanoparticles in AOT microemulsion system, Chem. Phys., 2006, 330, 495-500.

17 M. A. Raza, Z. Kanwal, A. Rauf, A. N. Sabri, S. Riaz and S. Naseem, Size-and shape-dependent antibacterial studies of silver nanoparticles synthesized by wet chemical routes, Nanomaterials, 2016, 6, 74. 
18 B. Sadeghi, F. S. Garmaroudi, M. Hashemi, H. R. Nezhad, A. Nasrollahi, S. Ardalan and S. Ardalan, Comparison of the anti-bacterial activity on the nanosilver shapes: nanoparticles, nanorods and nanoplates, Adv. Powder Technol., 2012, 23, 22-26.

19 H. Wang, X. Qiao, J. Chen and S. Ding, Preparation of silver nanoparticles by chemical reduction method, Colloids Surf., A, 2005, 256, 111-115.

20 R. M. El-Shishtawy, A. M. Asiri and M. M. Al-Otaibi, Synthesis and spectroscopic studies of stable aqueous dispersion of silver nanoparticles, Spectrochim. Acta, Part A, 2011, 79, 1505-1510.

21 O. Naderi, M. Nyman, M. Amiri and R. Sadeghi, Synthesis and characterization of silver nanoparticles in aqueous solutions of surface active imidazolium-based ionic liquids and traditional surfactants SDS and DTAB, J. Mol. Liq., 2019, 273, 645-652.

22 J. Hedberg, M. Lundin, T. Lowe, E. Blomberg, S. Wold and I. O. Wallinder, Interactions between surfactants and silver nanoparticles of varying charge, J. Colloid Interface Sci., 2012, 369, 193-201.

23 X. H. Vu, T. T. T. Duong, T. T. H. Pham, D. K. Trinh, X. H. Nguyen and V. S. Dang, Synthesis and study of silver nanoparticles for antibacterial activity against Escherichia coli and Staphylococcus aureus, Adv. Nat. Sci. Nanosci. Nanotechnol., 2018, 9, 025019.

24 S.-H. Kim, H.-S. Lee, D.-S. Ryu, S.-J. Choi and D.-S. Lee, Antibacterial activity of silver-nanoparticles against Staphylococcus aureus and Escherichia coli, Korean J. Microbiol. Biotechnol., 2011, 39, 77-85.

25 J. R. Morones, J. L. Elechiguerra, A. Camacho, K. Holt, J. B. Kouri, J. T. Ramírez and M. J. Yacaman, The bactericidal effect of silver nanoparticles, Nanotechnology, 2005, 16, 2346-2353.

26 M. R. Bindhu and M. Umadevi, Antibacterial and catalytic activities of green synthesized silver nanoparticles, Spectrochim. Acta, Part A, 2015, 135, 373-378.

27 D. Nayak, S. Pradhan, S. Ashe, P. R. Rauta and B. Nayak, Biologically synthesised silver nanoparticles from three diverse family of plant extracts and their anticancer activity against epidermoid A431 carcinoma, J. Colloid Interface Sci., 2015, 457, 329-338.

28 A. R. Allafchian, S. Z. Mirahmadi-Zare, S. A. H. Jalali, S. S. Hashemi and M. R. Vahabi, Green synthesis of silver nanoparticles using phlomis leaf extract and investigation of their antibacterial activity, J. Nanostruct. Chem., 2016, 6, 129-135.

29 P. Singh, Y. J. Kim and D. C. Yang, A strategic approach for rapid synthesis of gold and silver nanoparticles by Panax ginseng leaves, Artif. Cells Nanomed. Biotechnol., 2016, 44, 1949-1957.

30 M. M. O. Rashida, M. S. Islam, M. A. Haque, M. A. Rahman, M. T. Hossain and M. A. Hamid, Antibacterial activity of polyaniline coated silver nanoparticles synthesized from Piper betle leaves extract, Iranian J. Pharm. Res., 2016, 15, 591-597.

31 P. Prema, S. Thangapandiyan and G. Immanuel, CMC stabilized nano silver synthesis, characterization and its antibacterial and synergistic effect with broad spectrum antibiotics, Carbohydr. Polym., 2017, 158, 141-148.

32 M. Saravanan, S. K. Barik, D. MubarakAli, P. Prakash and A. Pugazhendhi, Synthesis of silver nanoparticles from Bacillus brevis (NCIM 2533) and their antibacterial activity against pathogenic bacteria, Microb. Pathog., 2018, 116, 221-226. 